\title{
Small Cell Neuroendocrine Carcinoma in Head and Neck
}

\author{
Sondang Nora Harahap*, Daan Khambri \\ Department of Surgical Oncology, DR. M. Djamil Hospital, Padang, Indonesia
}

\section{ARTICLE INFO}

Received : 16 November 2020

Reviewed : 24 March 2021

Accepted : 12 October 2021

\section{Keywords:}

head and neck cancer, immunohistochemistry, small cell neuroendocrine carcinoma

\author{
*Corresponding author: \\ Sondang Nora Harahap \\ Department of Surgical Oncology, \\ DR. M. Djamil Hospital, Padang, \\ Indonesia \\ sondangnora23@gmail.com
}

\begin{abstract}
Introduction: Poorly differentiated neuroendocrine carcinomas (NECs) originating from the eye are rare and very highly malignant diseases with a poor prognosis. Small cell NEC of the head and neck is a rare disease and highly aggressive. Early recognition and treatment are crucial for reducing morbidity and mortality.
\end{abstract}

Case Presentation: A 19-year-old male visited our oncology surgery outpatient department due to the progressive neck mass enlargement originating from the eye. The patient was previously diagnosed with invasive choroid malignant melanoma of the left eye which had metastasized to the lymph nodes of the left neck. He underwent a surgical removal/exenteration of the left eye. The result showed that the patient's survival with poorly differentiated tumors was about $14 \%$ while patients with well-differentiated NEC had a survival rate of $34 \%$. It also indicates that the prognosis of these tumors is very poor with a total of over $90 \%$ of patients having distant metastatic disease. Histopathological examination showed the tumor tissue and its immunohistochemistry with positive streaks of CD56, NSE, Synaptophysin, and Ki67 suggested small cell NEC.

Conclusions: it is crucial to establish an early diagnosis of these tumors to reduce morbidity and mortality. No optimal treatment for such disease has yet been established.

\section{INTRODUCTION}

Neuroendocrine carcinoma (NEC) is a composite tumor of the nervous and endocrine systems that can release neuropeptides into the systemic circulation. There have been some debates among pathologists about how to classify NECs of the head and neck. In 1993, the World Health Organization (WHO) classified laryngeal NEC into three categories: typical carcinoid, atypical carcinoid, and small cell carcinoma [1]. Small cell carcinoma of the head and neck is a rare disease and highly aggressive. It is associated with smoking and can occur throughout the head and neck region. The larynx, salivary glands, and the sinonasal region are the most common sites for small cell carcinoma of the head and neck [2]. Small cell NEC are highly aggressive malignancies that occur most commonly in the lung. Extrapulmonary small cell carcinomas comprise $2.5-5 \%$ of all small cell carcinomas [3].

Here, we report a case of rapid progression of small cell NEC in early young adults originating from the eye. Chemoradiation is a reasonable treatment for patients with small cell NEC [4].

\section{CASE PRESENTATION}

A 19-year-old male patient sought help due to the loss of vision of his left eye accompanied by left eye protrusion for the last year (Figure 1A). The patient was diagnosed with invasive choroid malignant melanoma of the left eye which had metastasized to the lymph nodes of the left neck. The definitive diagnosis was small cell NEC based on the histopathological examination and immunohistochemistry with a differential diagnosis of malignant lymphoma. He underwent a surgical removal/ exenteration of the left eye.

The difference between the two is that when using a definitive diagnosis, there will be a certainty of the results obtained. Histopathological examination showed the tumor tissue and its immunohistochemistry with positive streaks of CD56, NSE, Synaptophysin, and Ki67 suggestive of small cell NEC (Figure 2). The patient refused the chemotherapy with etoposide and cisplatin. The lump enlarged within 4 months and spread to the side of the left eye and neck (Figure 1B). He came to the oncology surgery with a very large lump on the head and neck. He complained of heavy and painful feelings, hoarseness, and impaired swallowing (Figure 
1C). The patient complained of a weight loss of $10 \mathrm{~kg}$ in one month. Another medical history was remarkable. There was no history of cigarette smoking and alcohol intake.

From the physical examination, he was found to have a Karnofsky score of 50 , a general state of serious illness to consciousness compos mentis cooperative, blood pressure of $110 / 70 \mathrm{mmHg}$, heart rate of $80 \mathrm{x}$ / $\mathrm{min}$, respiratory rate of $18 \mathrm{x} / \mathrm{min}$, a temperature of 36.5 degrees Celsius, and VAS 6. Based on the examination of the physical region of the head and neck, there was a large lump on the left side of the face starting from the left neck to the left temple measuring three times the size of the patient's face.
The lump appeared lumpy large with the surface of the skin partially shiny and ulcerated at the top of the lump, visible enlargement of the superficial veins, and the partly blackish skin. On palpation, there was a solid hard consistency, fixed, measuring $40 \times 30 \times 19 \mathrm{~cm}$. The intraoral examination found $1.5-\mathrm{cm}$ trismus and a protruding left buccal mass with mucous intake. On the examination of the right colli KGB, a lump was felt in the size of $7 \times 6 \times 4 \mathrm{~cm}$. Laboratory tests were performed, showing hemoglobin of $9.95 \mathrm{~g} / \mathrm{dL}$, leucocytes of $9,950 / \mathrm{mm}^{3}$, platelets of $337 / \mathrm{mm}^{3}$, hematocrit of $48 \%$, albumin of $3.5 \mathrm{~g} / \mathrm{dL}$, urea of $34 \mathrm{mg} / \mathrm{dL}$, blood creatinine of $0.9 \mathrm{mg} / \mathrm{dL}$, sodium of $136 \mathrm{mmol} / \mathrm{L}$, potassium of 4.3 $\mathrm{mmol} / \mathrm{L}$, and chloride $\mathrm{mmol} / \mathrm{L}$.
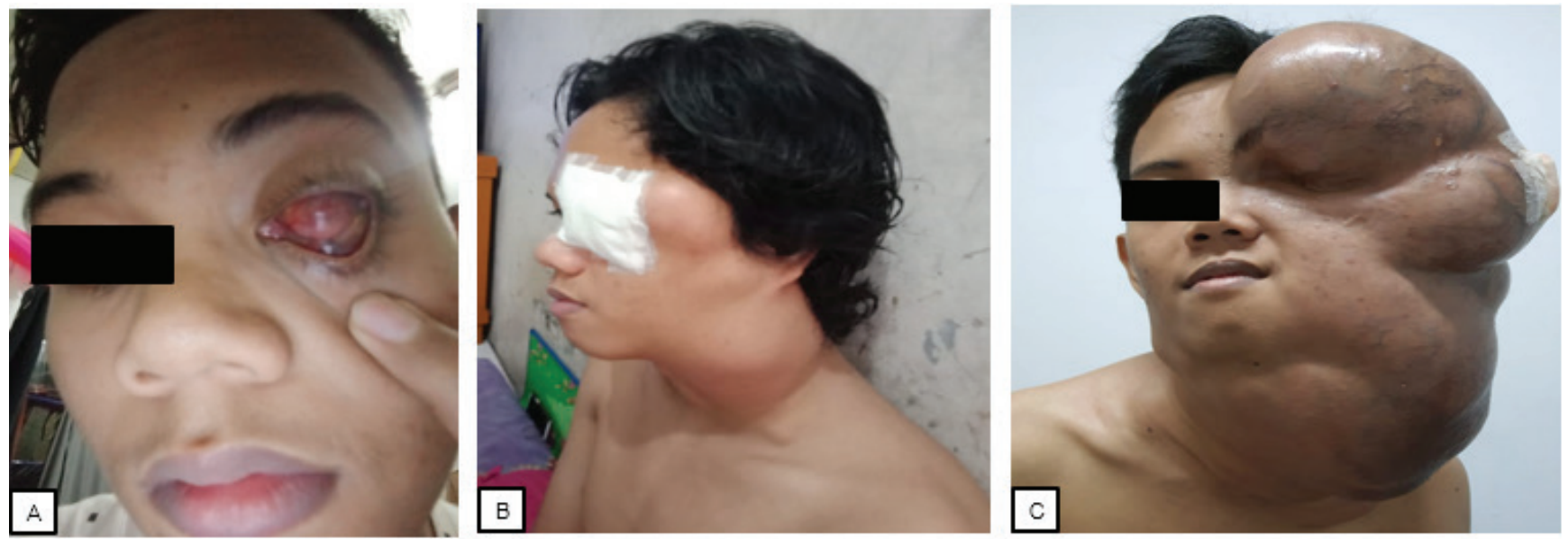

Figure 1. (A) Clinical features the first admission at Eye Department; (B) Progression 4 months later; (C) Clinical features the first admission at Surgical Oncology Department

Figure 2. Immunohistochemistry for small cell NEC $(\times 200)$.

(A) immune positive reaction for CD56; (B) immune positive reaction for NSE; (C) immune positive reaction for Ki-67; (D) immune positive reaction for Synaptophysin

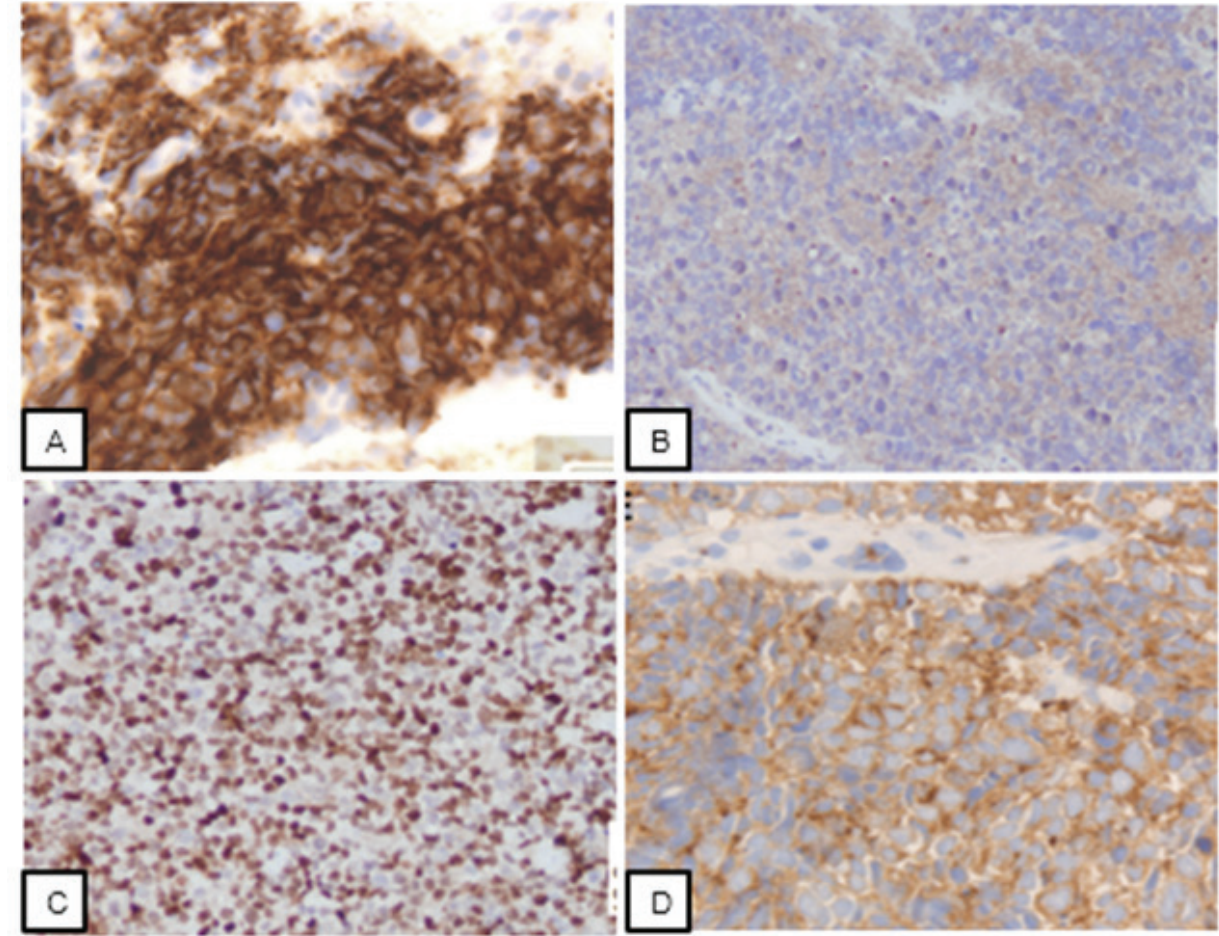




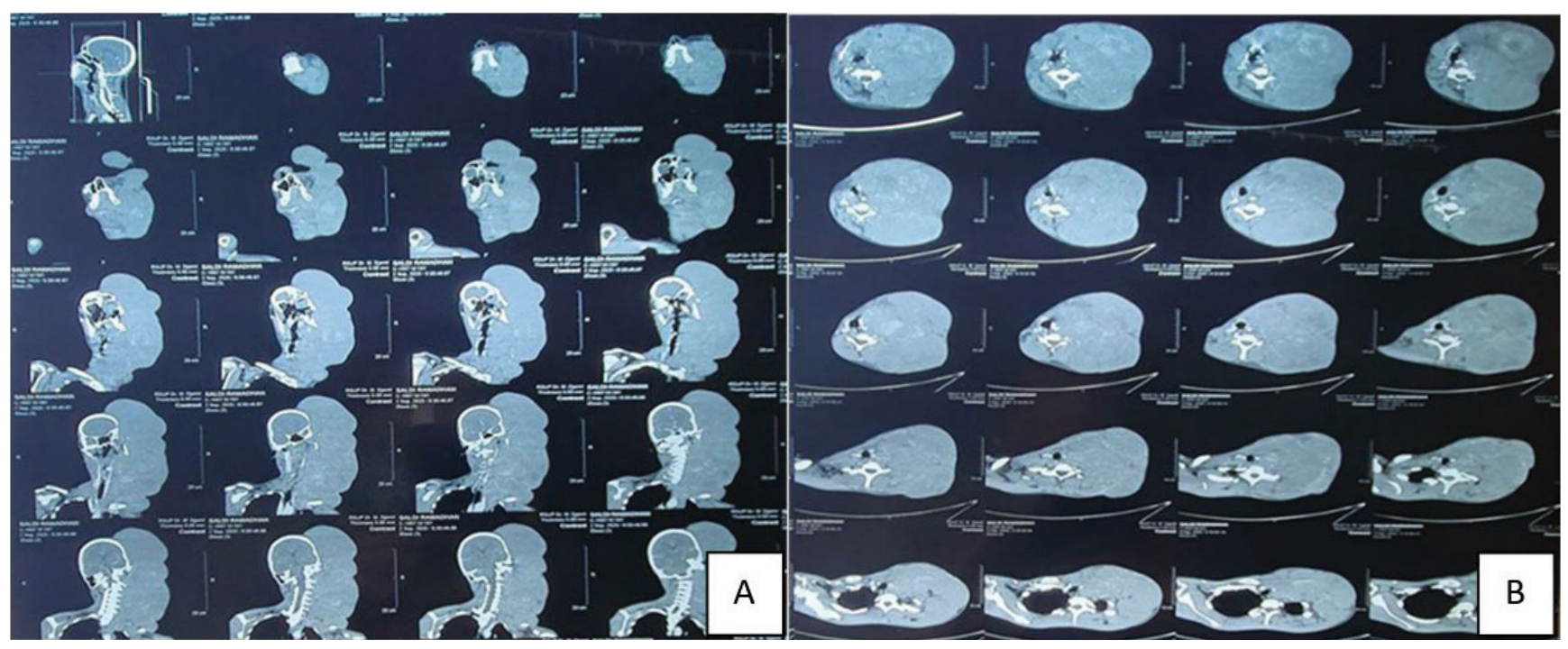

Figure 3. (A) a very large mass of inhomogeneous isodense density with a clearly defined lobulated edge of $37.8 \times 17.7 \times 20 \mathrm{~cm}$, accompanied by multiple calcifications from the frontal to the left superior mediastinum; (B) The mass appears to provide light enhancement on intravenous contrast administration. The mass appears to extend to the frontal sinus, the left orbital extending to the left ethmoid sinus, left maxillary sinus, left nasal cavity, nasopharynx. The larynx is pushed to the right.

On the CT scan of the head and neck with contrast (Figure 3), a very large mass of inhomogeneous isodense density was found with a distinct lobulated edge measuring $37.8 \times 17.7 \times 20 \mathrm{~cm}$ accompanied by multiple calcifications from the frontal to the left superior mediastinum. The mass appeared to enhance lightly on the intravenous contrast administration. The mass appeared to extend to the frontal sinus, the left orbital extending to the left ethmoid sinus, left maxillary sinus, left nasal cavity, and nasopharynx. The larynx was pushed to the right. Intra cerebral was within normal limits. In the Thoracic CT scan, there was no picture of metastases. On the abdominal ultrasound examination, there was no picture of metastasis to the liver and other intraabdominal organs.

The patient was diagnosed based on the histopathological examination and immunohistochemistry with small cell NEC T4bN3bM0. The patient was unresectable with a severe general condition accompanied by the possibility of tumor compression in the trachea and esophagus. He was managed for general condition improvement by providing adequate nutritional intake via a nasogastric tube, and gastrostomy might be done if the nasogastric tube was not possible to continue with preparation for palliative chemotherapy. If the tumor size was reduced, radiation was performed. The patient's condition decreased and the lumps in the head and neck were getting bigger rapidly, especially towards the left hemithorax. In preparation for chemotherapy, the patient was declared dead.

\section{DISCUSSION}

NEC is a composite tumor of the nervous and endocrine systems that can release neuropeptides into the systemic circulation. 1 Small cell carcinoma histopathologically is a malignant epithelial tumor consisting of small cells with little cytoplasm, fine granular nucleus chromatin, unclear cell boundaries, and absent or inconspicuous nucleoli. More than $90 \%$ of small cell carcinomas have neuroendocrine features [4].

The WHO has characterized them into four subtypes of neuroendocrine: typical carcinoid tumors, atypical carcinoid tumors, small cell neuroendocrine tumors, and paragangliomas [5]. Some risk factors reported were age > 50-65 years, smoking, male gender, history of alcohol consumption, and history of radiation. However, these risk factors do not cause causative small cell NEC. In this patient, no risk factors were found, except gender [6]. Symptoms generally depend on the specific tumor site. Dysphagia, a globus sensation, and respiratory distress can occur with laryngeal lesions. Pain or a palpable mass suggests a diagnosis of salivary gland involvement. Facial nerve palsy is a more ominous sign that typically identifies a malignant rather than benign disease [7].

Conventional anatomical imaging and functional imaging using radionuclide scintigraphy and positron emission tomography/computed tomography can be complementary for the diagnosis, staging, and monitoring of treatment response. Multislice CT facilitates rapid 
and detailed evaluation of the entire neck with the ability to produce multiplanar reformatted images. MRI has a superior soft-tissue resolution, which makes it an ideal technique for imaging head and neck masses. It is superior to $\mathrm{CT}$ in defining the intracranial extension of tumors [8].

NECs are classified with their mitotic count and Ki67 index. The mitotic counts of poorly differentiated NECs, generally called high-grade or G3 NECs, are greater than $20 \times 10 \mathrm{HPFs}$, and the Ki-67 level is greater than $20 \%$. The angioinvasion of high-proliferation tumors with a Ki-67 level higher than $20 \%$ is extensive, and these tumors demonstrate an incredible potential to create metastatic disease [9]. In concordance with the Ki-67 positive result, this patient showed rapid progression and metastases.

There is no consensus on the treatment of NEC. The treatment of extrapulmonary neuroendocrine tumors depends on whether the tumor is resectable, locoregionally advanced but unresectable, or metastatic [10]. Surgical operation is not always the first choice due to the postoperative quality of life impairment. Combined chemoradiotherapy aiming at organ preservation is preferred. Based on the results of the multidisciplinary tumor board meeting, chemotherapy and radiation preparations are still being carried out because there is no airway obstruction in the hope that the tumor size will decrease so that it makes it easier to carry out tracheostomy and other surgical interventions [11].

Deglutition has been severely impaired due to tumor compression to the trachea and esophagus in this patient. The primary lesion was unknown, and appropriate surgery was possible because the metastases occurred in one organ at a time [11]. Radical surgery needs to be implemented rather than chemoradiotherapy. Kuan et al. divided NEC of the head and neck into sinonasal and non-sinonasal, according to the site of the primary tumor. They reported that patients with sinonasal primary tumors experienced improved survival with surgery while those with non-sinonasal tumors had better survival with radiation therapy [12]. There is a higher risk of failed completion of chemoradiotherapy in patients whose deglutition has been severely compromised by advanced hypopharyngeal tumors [13].

Unfortunately, the general condition was not appropriate for surgery and the patient's condition continued to deteriorate. The survival rates of the patients with head/neck NEC vary somewhat but are poor overall. The five-year survival of patients with poorly differentiated tumors is $14 \%$ while patients with well-differentiated NEC have a 5-year survival rate of $34 \% .7$ The prognosis of these tumors is very poor with over $90 \%$ of patients developing the distant metastatic disease [14].

\section{CONCLUSIONS}

It is crucial to establish an early diagnosis of these tumors as they can reduce morbidity and mortality. No optimal treatment for such disease has yet been established. It is because this case has never been found in our hospital so that the management follows the results of the tumor board meeting.

\section{DECLARATIONS}

\section{Ethics of Approval Consent to Participate NA}

\section{Competing of Interest}

The authors declare no competing interest in this study.

\section{Acknowledgment}

NA

\section{REFERENCES}

1. Meacham R, Matrka L, Ozer E, et al. Neuroendocrine carcinoma of the head and neck: A 20-year case series. Ear Nose Throat J. 2012;91(3):20-4.

2. Pointer KB, Ko HC, Brower JV, et al. Small cell carcinoma of the head and neck: An analysis of the National Cancer Database. Oral Oncol. 2017;69:92-8.

3. Iqbal MS, Paleri V, Moor J, et al. Small cell neuroendocrine carcinoma of larynx: Case series and literature review. J Laryngol Otol. 2015;129(9):910-5.

4. Hosokawa S, Takahashi G, Baba S, Mineta H. Small cell neuroendocrine carcinomas arising in the head and neck region. J Oral Maxillofac Surg. 2016;74(5):1091-5.

5. Fisseler-Eckhoff A, Demes M. Neuroendocrine tumors of the lung. Cancers (Basel). 2012; 4(3):777-98.

6. Ao YJ, Zhou SH. Primary poorly differentiated small cell type neuroendocrine carcinoma of the hypopharynx. Onco Targets Ther. 2019;12:1593-601.

7. Scaduto M, Matrka L, Shah M, et al. Poorly differentiated small-cell neuroendocrine carcinoma of the submandibular gland: a case report. Ear Nose Throat J. 2012;91(4):E10-3.

8. Subedi N, Prestwich R, Chowdhury F, et al. Neuroendocrine tumours of the head and neck: Anatomical, functional and molecular imaging and contemporary management. Cancer Imaging. 2013;13(3):407-22.

9. Nadler A, Cukier M, Rowsell C, et al. Ki-67 is a reliable pathological grading marker for neuroendocrine tumors. Virchows Arch. 2013;462(5):501-5. 
10. van der Laan TP, Plaat BE, van der Laan BF, Halmos $G B$. Clinical recommendations on the treatment of neuroendocrine carcinoma of the larynx: A metaanalysis of 436 reported cases. Head Neck. 2015;37(5):707-15.

11. Takagawa R, Tanaka K, Yamada $M$, et al. Primary neuroendocrine carcinoma of the hypopharynx: a case report. Dis Esophagus. 2011;24(3):E26-E31.

12. Kuan EC, Alonso JE, Tajudeen BA, et al. Small cell carcinoma of the head and neck: A comparative study by primary site based on population data. Laryngoscope. 2017;127(8):1785-90.
13. Nakahira M, Kuba K, Matsumura S, Sugasawa M. Primary small cell carcinoma of the hypopharynx: a report of two cases and review of nine additional cases. Case Rep Otolaryngol. 2017;2017:1-7.

14. Lee JE, Hong $\mathrm{SH}$, Jung $\mathrm{HI}$, et al. Small-cell neuroendocrine carcinoma of the ileum: Case report and literature review. BMC Surg. 2019;19(1):1-5. 